\title{
Remote Electrical Neuromodulation for the Acute Treatment of Migraine in Patients with Chronic Migraine: An Open-Label Pilot Study
}

\author{
Hida Nierenburg · Julio R. Vieira • Nirit Lev • Tamar Lin · \\ Dagan Harris · Maya Vizel · Alon Ironi · Bryan Lewis • \\ Paul Wright
}

Received: May 11, 2020 / Published online: July 9, 2020

(C) The Author(s) 2020

\section{ABSTRACT}

Introduction: Remote electrical neuromodulation (REN) is a novel acute treatment of migraine. Upper arm peripheral nerves are stimulated to induce conditioned pain modulation (CPM) - an endogenous analgesic mechanism in which conditioning stimulation inhibits pain in remote body regions. The REN device $\left(\right.$ Nerivio $^{\circledR}$, Theranica Bio-Electronics LTD., Israel) is FDA-authorized for acute treatment of migraine in adults who do not have chronic migraine. The current study assessed

Digital Features To view digital features for this article go to https://doi.org/10.6084/m9.figshare.12589455.

H. Nierenburg $(\bowtie) \cdot J$. R. Vieira · B. Lewis .

P. Wright

Division of Neurology, Nuvance Health,

Poughkeepsie, NY, USA

e-mail: hida.nierenburg@nuvancehealth.org

J. R. Vieira

Albert Einstein College of Medicine, The Saul R.

Korey Department of Neurology, Bronx, NY, USA

N. Lev

Department of Neurology, Meir Medical Center, Kfar Saba, Israel

N. Lev

Sackler Faculty of Medicine, Tel Aviv University, Tel

Aviv, Israel

T. Lin · D. Harris · M. Vizel · A. Ironi

Theranica Bio-Electronics, Netanya, Israel the consistency of response over multiple migraine attacks in people with chronic migraine who are typically characterized with severe pain intensity, high disability, and less robust response to triptans.

Methods: This was an open-label, single-arm, dual-center study conducted on adults with chronic migraine. Participants underwent a 4-week treatment phase in which they treated their migraine headaches with the device for 45 min within $1 \mathrm{~h}$ of attack onset. Pain levels were recorded at baseline, $2 \mathrm{~h}$, and $24 \mathrm{~h}$ posttreatment. Efficacy outcomes (pain relief and pain-free responses at $2 \mathrm{~h}$, sustained pain relief and sustained pain-free responses at $24 \mathrm{~h}$ ) focused on intra-individual consistency of response across multiple attacks, which was defined as response in at least $50 \%$ of the treatments.

Results: Forty-two participants were enrolled, and 38 participants were evaluable for analyses; $73.7 \%(28 / 38)$ achieved pain relief at $2 \mathrm{~h}, 26.3 \%$ $(10 / 38)$ were pain-free at $2 \mathrm{~h}, 84.4 \%(27 / 32) \mathrm{had}$ sustained pain relief response at $24 \mathrm{~h}$ and $45.0 \%$ $(9 / 20)$ had sustained pain relief response at $24 \mathrm{~h}$ in at least $50 \%$ of their treated attacks. The effects of REN on associated symptoms and improvement in function were also consistent. The incidence of device-related adverse events was low (1.8\%).

Conclusions: REN used for a series of migraine attacks was effective and well tolerated across attacks. REN may offer a safe and effective non- 
pharmacological alternative for acute treatment in patients with chronic migraine.

Trial Registration: ClinicalTrials.gov identifier, NCT04161807. Retrospectively registered on November 13, 2019.

Keywords: Acute migraine treatment; Chronic migraine; Consistency; Headache; Remote electrical neuromodulation; Medication overuse headache; Nerivio

\section{Key Summary Points}

Chronic migraine is a disabling neurological condition that affects $2 \%$ of the general population.

Current acute treatments of migraine may be ineffective, poorly tolerated, contraindicated, and if used in excess, may lead to medication overuse headache.

Remote electrical neuromodulation (REN) is a novel non-pharmacological acute treatment of migraine applied to the upper arm.

REN used for a series of migraine attacks was effective and well tolerated across attacks, and may offer an alternative acute therapy which may reduce medication use and thus reduce the risk for medication overuse headache.

\section{INTRODUCTION}

Chronic migraine is a disabling neurological condition that affects $2 \%$ of the general population [1]. Patients with chronic migraine have headaches on at least 15 days a month, with at least eight days a month on which their headaches and associated symptoms meet diagnostic criteria for migraine. Typically, chronic migraine develops after a slow increase in headache frequency over months to years, in a process termed migraine chronification. The risk of developing chronic migraine mainly depends on the frequency with which acute medications are used and the type of medication used [2].

The main goals of treatment of chronic migraine are to reduce the frequency of migraine attacks and to reduce migraine-related disability while avoiding the overuse of migraine acute medications [2]. Currently, the first line of acute treatment of migraine is pharmacological. Acute treatment options typically include simple and combination analgesics, nonsteroidal anti-inflammatory drugs (NSAIDs), triptans [3], lasmiditan [4], and gepants (ubrogepant and rimegepants) $[5,6]$. However, the frequency of migraine acute medication use must be limited $(<10$ days per month of migraine-specific medications) to avoid overuse and medication overuse headache $(\mathrm{MOH})$, which manifests as an increase in the frequency and intensity of headaches $[7,8]$. This limitation introduces a great unmet need for alternative non-pharmacological acute migraine treatments that are effective and well tolerated. Such developments hold the potential to improve the health and quality of life of patients with chronic migraine.

Non-invasive neuromodulation devices represent an emerging field in the treatment of headache in general and migraine in specific [9-15]. Remote electrical neuromodulation (REN) [16-20] is a non-pharmacological, noninvasive, acute migraine treatment that stimulates upper arm peripheral nerves to induce conditioned pain modulation (CPM) -an endogenous analgesic mechanism in which a conditioning stimulation inhibits pain in remote body regions [21]. The REN device (Nerivio ${ }^{\circledR}$, Theranica Bio-Electronics Ltd., Israel) is a wireless, wearable, battery-operated stimulation unit controlled by a smartphone software application. The device is applied for $45 \mathrm{~min}$ to the lateral upper arm and mainly stimulates small skin nerves.

The safety and efficacy of REN have been previously assessed for migraine in a randomized, double-blind, sham-controlled multi-center study [17]. This study demonstrated that REN provides superior clinically meaningful relief of migraine pain and most bothersome symptom (MBS) compared to placebo, offering a 
safe and effective non-pharmacological alternative for acute migraine treatment. Since this study was conducted according to the International Headache Society guidelines for acute treatment of migraine trials in adults [22], chronic migraine patients were excluded, as typically done in migraine acute treatment studies [4, 23, 24]. The current study assessed the safety and efficacy of Nerivio specifically in people with chronic migraine. We specifically focused on intra-individual consistency of pain response across multiple attacks. Demonstrating consistency of a treatment is clinically important especially in a chronic migraine population which experiences numerous attacks per month, as it would indicate that a treatment can be relied on by the patients, which can improve adherence, reduce migraine-related disability, reduce medication overuse, and increase overall confidence in efficacy [25].

\section{METHODS}

\section{Standard Protocol Approvals, Registrations, and Patient Consents}

The study was conducted at one site in the USA and one site in Israel. The study protocol was reviewed and approved by the local IRB of Meir Medical Center and Vassar Brothers Medical Center IRB and was conducted according to Good Clinical Practice and the Declaration of Helsinki guidelines. Before undergoing any study procedures, patients provided written informed consent. The first patient was enrolled in September 2019, and the last patient completed the treatment phase of the study in February 2020. The study is registered with ClinicalTrials.gov (NCT04161807).

\section{Study Design and Participants}

Patients with chronic migraine participated in this prospective, open-label, single-arm, dualcenter study conducted at one clinic in the USA and one site in Israel. Patients were eligible to participate if they were 18-75 years old, met the
International Classification of Headache Disorders (ICHD-3) criteria for chronic migraine (at least 15 headache days a month, with at least 8 days a month on which their headaches and associated symptoms meet diagnostic criteria for migraine) [26], and were on either no, or stable migraine-preventive medications in the last 2 months prior to recruitment. Exclusion criteria were: (1) pregnancy, nursing, trying to conceive; (2) implanted electrical and/or neurostimulator device; (3) congestive heart failure (CHF), severe cardiac or cerebrovascular disease; (4) uncontrolled epilepsy; (5) lack of efficacy, after an adequate therapeutic trial, of at least two migraine-specific acute medications; (6) other significant pain, medical or psychiatric illness that in the opinion of the investigator may confound the study assessments; (7) unable to use a smartphone; (8) previous experience with Nerivio; (9) participating in any other interventional clinical study.

\section{The REN Device}

Nerivio is a wireless, wearable, non-invasive stimulation device applied to the lateral upper arm between the bellies of the lateral deltoid and the triceps for $45 \mathrm{~min}$ (Fig. 1). The device stimulates small skin nerves using a proprietary electrical signal comprising a modulated, symmetrical, biphasic, square pulse with a modulated frequency of 100-120 Hz, pulse width of $400 \mu \mathrm{s}$, and up to $40 \mathrm{~mA}$ output current (adjusted by the patient). The pulse is designed to stimulate $\mathrm{C}$ and $\mathrm{A} \delta$ noxious sensory fibers above their depolarization thresholds, yet the stimulation energy is low enough to maintain the overall sensory experience below the perceptual pain threshold. Since REN induces a global pain inhibition mechanism in which pain in the arm inhibits pain in other areas of the body, the device can be used on either arm independently from the side of a unilateral headache. The efficacy of the treatment does not depend on which arm the device is applied to. Nerivio is controlled by an app that includes a migraine diary to track treatment sessions and migraine headaches. 


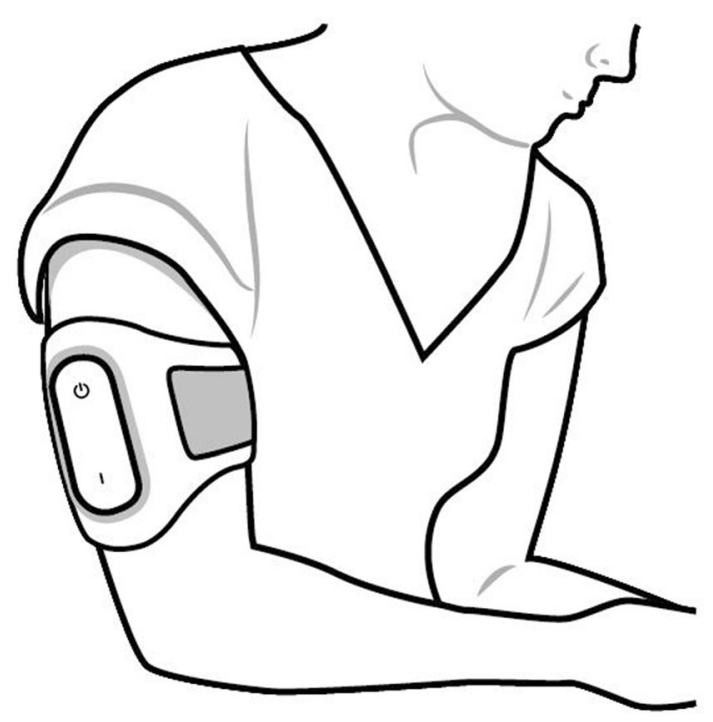

Fig. 1 Illustration of the REN device

\section{Procedures}

During the enrollment visit, participants were introduced with the device and trained to use it. Specifically, an optimal individual stimulation intensity level just below the pain threshold was identified for each participant. The individualized intensity should be perceptible but not painful, so it will be strong enough to activate $\mathrm{C}$ and $A \delta$ noxious sensory fibers to induce the CPM without causing pain in the arm. Participants were also trained how to record the symptoms in an electronic migraine diary. Participants treated their migraine attacks at home for 4 weeks (treatment phase), with their optimal stimulation intensity. Participants were instructed to treat all their migraine headaches as soon as possible after migraine headache began and always within $1 \mathrm{~h}$ of symptom onset, and to avoid taking rescue medications within $2 \mathrm{~h}$ post-treatment. Pain scores (none, mild, moderate, or severe), absence or presence of associated symptoms (nausea and/or vomiting, photophobia, and phonophobia) and functional disability (no limitation, some limitation, moderate limitation, severe limitation) were recorded at baseline, at $2 \mathrm{~h}$ post-treatment, and at $24 \mathrm{~h}$ post-treatment in an electronic diary application installed on the participants' smartphones.

\section{Outcomes}

Efficacy outcomes focused on intra-individual consistency of response across multiple attacks. A consistent response was defined by efficacy in at least $50 \%$ of the treated attacks. This approach of consistency assessment has an important advantage of using all available data and enables the inclusion of all patients in the analyses. The pain response endpoints include consistency of pain relief response (defined as a decrease in headache pain from moderate or severe at baseline to mild or no pain at $2 \mathrm{~h}$ after treatment or a decrease in headache pain from mild at baseline to no pain at $2 \mathrm{~h}$ after treatment); consistency of pain-free response (defined as a decrease in headache pain from mild, moderate, or severe at baseline to no pain at $2 \mathrm{~h}$ after treatment); consistency of sustained pain relief response (defined as mild or no pain at $24 \mathrm{~h}$ without medication within $24 \mathrm{~h}$ of treatment start, in treatments in which pain relief was achieved at $2 \mathrm{~h}$ ); and consistency of sustained pain-free response (defined as no pain at $24 \mathrm{~h}$ without medication within $24 \mathrm{~h}$ of treatment start in treatments in which pain freedom was achieved at $2 \mathrm{~h}$ ). Additional efficacy endpoints include consistency of disappearance of nausea and/or vomiting, consistency of disappearance of photophobia, and consistency of disappearance of phonophobia, consistency of improvement in function (defined as improvement in at least one grade at $2 \mathrm{~h}$ ), and consistency of return to normal function (defined as no functional disability at $2 \mathrm{~h}$ ).

\section{Data Analysis}

The intention-to-treat (ITT) population included all participants who received the device and was used for the efficacy and safety analyses. The challenge with acute treatment in chronic migraine patients is to avoid treatments of recurrent headaches in a study setting. The International Headache Society guidelines for acute treatment of migraine trials in adults require that the analyses will be conducted on treated attacks preceded by at least 48 headache-free hours. This restriction is applicable for 
people without chronic migraine but cannot be applied to a study conducted on a chronic population with over 15 headache days per month. Therefore, for the purpose of this study, a qualifying migraine headache was defined as a migraine headache that is of moderate or severe intensity and preceded by $24 \mathrm{~h}$ of no pain or mild intensity pain; or is of mild intensity and preceded by $24 \mathrm{~h}$ of no pain. Although participants were instructed to treat all headaches, only qualifying headaches were included in the efficacy analyses to avoid assessing efficacy on recurrent headaches.

The first reported treatment of each participant was considered a training treatment and was only included in the safety analyses. Within-subject consistency across multiple attacks was based on all fully treated (30-45 min) qualifying migraine headaches with baseline and 2-h post-treatment assessment following the training treatment. Use of rescue medication before the 2 -h assessment was considered a treatment failure. To estimate the long-term response to the treatment, painrelief and pain-free responses were analyzed across the first five consecutive evaluable treatments within each participant. The analyses assessing the consistency of sustained pain response were conducted on treatments in which pain level and medication use were reported at $24 \mathrm{~h}$. Treatments with missing data were excluded from all analyses.

For the associated symptoms outcomes, patients with presence of a symptom at baseline and data at $2 \mathrm{~h}$ are included in the analyses. A response in each associated symptom (nausea/ vomiting, photophobia, phonophobia) is defined as change from presence of a specific associated symptom at baseline to absence of the same associated symptom at $2 \mathrm{~h}$ post-treatment. For functional disability endpoints, all patients with baseline values of "some limitation", "moderate limitation", or "severe limitation", and data at $2 \mathrm{~h}$ were included in the analyses. Improvement in function is defined as a reduction of at least one grade at $2 \mathrm{~h}$ posttreatment and return to normal function is defined as "no limitation" at $2 \mathrm{~h}$.

For continuous variables, mean and standard deviation or median and IQR were provided. For categorical variables, the number and percentage of patients in each category are provided with $95 \%$ confidence intervals. Data were analyzed with IBM SPSS statistics software version 25.0. (SPSS Inc., USA).

\section{RESULTS}

\section{Participants}

This study was conducted from September 18, 2019 to February 18, 2020. Forty-two patients were enrolled and received a device (Fig. 2). Two participants withdrew from the study; one since the protocol requirements were too time consuming for him and one due to treatment intolerability. All participants completed at least one treatment (the training treatment) and 38 participants completed at least one additional treatment with baseline and $2-\mathrm{h}$ data following the training treatment, forming the final analysis set (one participant only had migraine headaches presented upon awakening which were not treated since the time of onset was unknown, and one participant decided not to treat).

The demographic and clinical characteristics of the participants (Table 1) and the characteristics of treated migraine headaches were comparable to those reported in epidemiological studies of migraine [27]. Furthermore, the clinical characteristics of the participants correspond with a chronic migraine diagnosis according to the ICHD-3 criteria [26].

\section{Treated Migraine Headaches}

A total of 296 qualifying migraine headaches were treated with REN (average of 7.0 REN treatments per participant per 4 weeks for 42 participants), of which baseline pain level was reported in 270 treatments. Pain severity of treated migraine headaches was mostly moderate $[54.1 \%(146 / 270)]$ and $48 / 270(17.8 \%)$ of the treated migraine headaches were severe and $76 / 270(28.2 \%)$ of the treated migraine headaches were mild. A total of 210 evaluable treatments (excluding the training treatment) of 


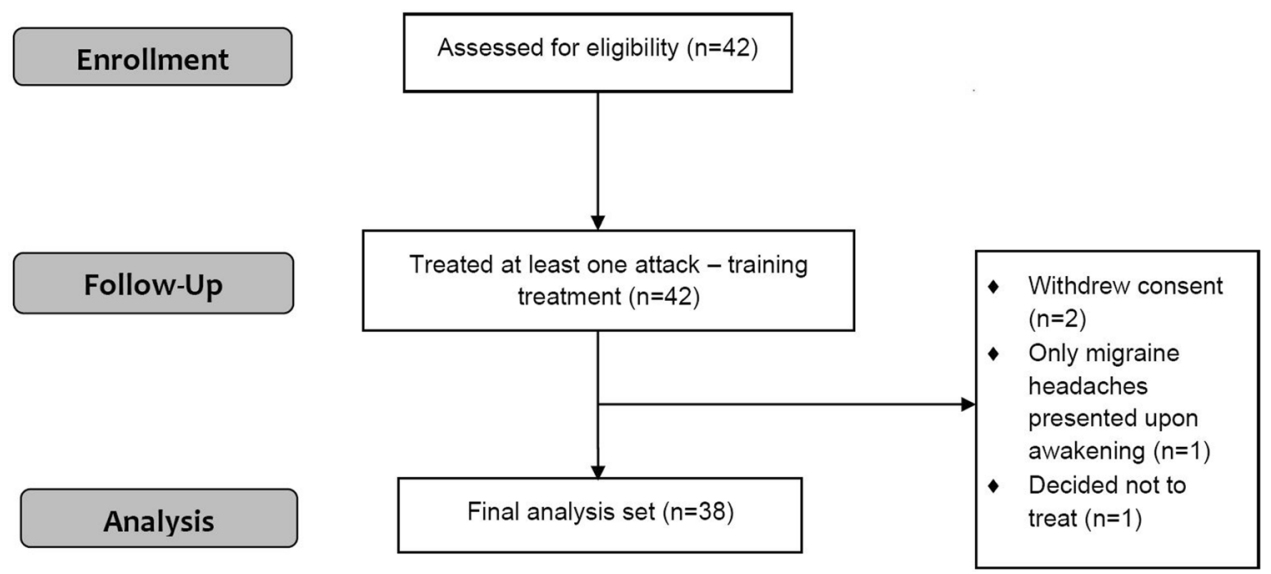

Fig. 2 Disposition of participants

Table 1 Demographic and clinical characteristics (intent-to-treat population)

\begin{tabular}{lc}
\hline Characteristic & ITT \\
\hline Age, years $(S D)$ & $42.1(14.8)$ \\
Female, $\%(n / N)$ & $83.3 \%(35 / 42)$ \\
Caucasian, $\%(n / N)$ & $100 \%(42 / 42)$ \\
Average number of headache days per month & $21.5(6.3)$ \\
Average number of migraine headache das per month & $18.3(6.6)$ \\
Triptan users, $\%(n / N)$ & $69.0 \%(29 / 42)$ \\
Migraine with aura, $\%(n / N)$ & $23.8 \%(10 / 42)$ \\
MBS $\%(n / N)$ & $57.2 \%(24 / 42)$ \\
Nausea & $30.9 \%(13 / 42)$ \\
Photophobia & $9.5 \%(4 / 42)$ \\
Phonophobia & $2.4 \%(1 / 42)$ \\
Allodynia & $64.3 \%(27 / 42)$ \\
Preventive medication use, $\%(n / N)$ & 296 \\
Treated qualifying migraine headaches in the treatment phase, $n$ & \\
\hline
\end{tabular}

qualifying migraine headaches were conducted by the 38 participants included in the analyses, with an average of $5.5 \pm 2.6$ evaluable treatments per patient per 4 weeks. Baseline pain level was moderate-to-severe in more than half of the treated attacks in 29 of the 38 patients included in the analyses (76.3\%).

Medication at $2 \mathrm{~h}$ was used in 22 of the 210 treatments (89.5\% compliance rate); $57.9 \%$ (22/ 38 ) of the patients did not use medication at $2 \mathrm{~h}$ for all their treatments during the study. 


\section{Efficacy Outcomes}

Consistency analyses across all attacks (excluding the training treatment) demonstrated that $73.7 \%\left(28 / 38\right.$; $\left.\mathrm{CI}_{95 \%} 60.0-87.4 \%\right)$ of the participants experienced pain relief in at least $50 \%$ of their treated attacks, and $26.3 \%\left(10 / 38 ; \mathrm{CI}_{95 \%}\right.$ $13.4-43.1 \%)$ of the participants experienced pain-free in at least $50 \%$ of their treated attacks (Fig. 3a and Table 2). At $2 \mathrm{~h}$, mean pain relief rate across subjects was $59.3 \%$, median pain relief rate across subjects was $60 \%$, and the inter quartiles range (IQR) was 43.67-87.50\%. Mean pain-free rate across subjects was $25.9 \%$, median pain-free rate across subjects was $20 \%$ and the IQR was $0-50 \%$.

To derive more stable estimates of long-term response to the treatment at $2 \mathrm{~h}$, pain-relief and pain-free responses were analyzed across the first five consecutives evaluable treatments (including the training treatment) within each participant. For each attack considered separately, pain relief and pain-free responses at $2 \mathrm{~h}$ post-treatment ranged from 50.0 to $63.3 \%$ and 23.3 to $27.0 \%$, respectively (Fig. 4; Tables 3,4 ).

Across all attacks, $84.4 \% \quad\left(27 / 32 ; \quad \mathrm{CI}_{95 \%}\right.$ $71.8-97.0 \%)$ and $45.0 \% \quad\left(9 / 20 ; \quad \mathrm{CI}_{95 \%}\right.$ 23.2-66.8\%) of patients reported sustained pain relief and sustained pain-free responses at $24 \mathrm{~h}$ in at least $50 \%$ of their treated attacks, respectively (Fig. 3b and Table 2). For the associated symptoms, $58.3 \%\left(14 / 24 ; \mathrm{CI}_{95 \%} 38.6-78.1 \%\right)$, $35.5 \%\left(11 / 31 ; \mathrm{CI}_{95 \%} 18.6-52.3 \%\right)$ and $40.0 \%$ (10/25; $\mathrm{CI}_{95 \%}$ 20.8-59.2\%) of patients experienced disappearance of nausea and/or vomiting, disappearance of photophobia, and disappearance of phonophobia at $2 \mathrm{~h}$ in at least $50 \%$ of their treated attacks, respectively. Furthermore, $45.7 \%\left(16 / 35 ; \mathrm{CI}_{95 \%} 29.2-62.2 \%\right)$ and $28.6 \%\left(10 / 35 ; \mathrm{CI}_{95 \%} 13.6-43.5 \%\right)$ of patients reported improvement in function and return to normal function at $2 \mathrm{~h}$ in at least $50 \%$ of their attacks, respectively (Table 2 ).

\section{Safety}

Safety analyses were performed on all 42 participants who used the device at least once (ITT population). The percentage of participants experiencing at least one adverse event was $4.7 \%\left(2 / 42 ; \mathrm{CI}_{95 \%} 0.6-16.2 \%\right)$. One device-related adverse event was reported [1.8\% (1/42)]. This adverse event included bilateral tingling in the temples and disturbed and double vision. The device-related adverse event was moderate and resolved within $48 \mathrm{~h}$ following drug therapy (triptan). There were no device-related serious adverse events and none of the participants withdrew from the study due to devicerelated adverse events.

\section{DISCUSSION}

This study demonstrates that REN may offer an efficacious treatment option with a favorable safety profile for acute treatment of migraine in patients with chronic migraine and extends previous studies establishing the efficacy of REN in patients with non-chronic migraine $[17,20]$. Overall, the data reveal consistent response rates from treatment to treatment, with no evidence of reduction in therapeutic benefits over time. Specifically, over $73 \%$ of the patients achieved pain relief at $2 \mathrm{~h}$ in more than half of their attacks and over $26 \%$ of the patients achieved pain freedom at $2 \mathrm{~h}$ in most attacks. Our findings also demonstrate a consistent sustained therapeutic response; approximately $85 \%$ of participants had sustained pain relief at $24 \mathrm{~h}$ in more than half of their attacks and $45 \%$ had a consistent sustained pain-free response. These findings suggest that REN may address patients' desire for a migraine therapy that provides consistent efficacy across multiple attacks [28].

REN likely utilizes a descending endogenous analgesic mechanism to inhibit migraine pain. While the descending pain modulatory system is well characterized [29], this natural mechanism has not been utilized for non-pharmacological pain treatments, except for invasive spinal cord stimulation that is believed to activate this system [30]. Engagement of this descending network is believed to induce CPM [31] (a human parallel of the animal lab-based diffuse noxious inhibitory control effect [32]) to inhibit headache pain by peripheral nociceptive information that is below the perceived pain 
A

Pain response at 2 hours

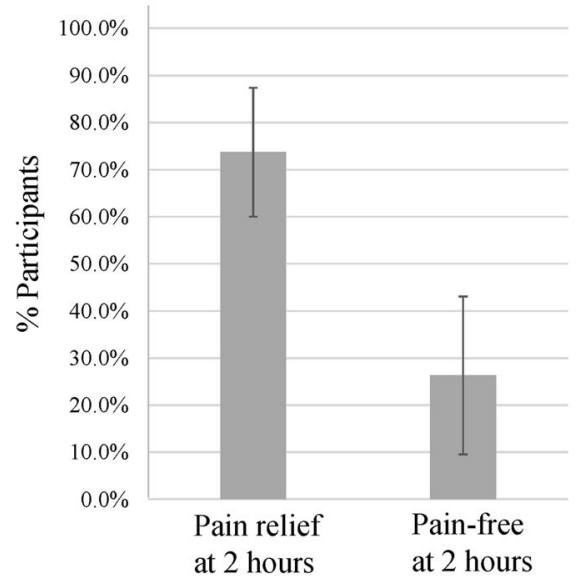

Fig. 3 Consistency of pain response. a Percentage of participants achieving pain relief and pain-free at $2 \mathrm{~h}$ response in at least $50 \%$ of treated attacks. b Percentage of participants achieving sustained pain relief at $24 \mathrm{~h}$ and

B Sustained pain response at 24 hours

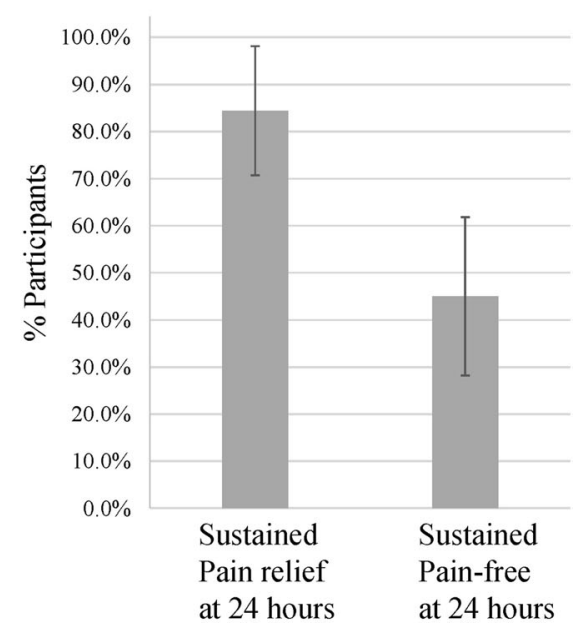

sustained pain-free response in at least $50 \%$ of treated attacks. The error bars represent $95 \%$ confidence intervals

Table 2 Efficacy outcomes

Consistency of pain-relief response at $2 \mathrm{~h}$

Consistency of pain-free response at $2 \mathrm{~h}$

Consistency of sustained pain-relief response at $24 \mathrm{~h}$

Consistency of sustained pain-free response at $24 \mathrm{~h}$

Consistency of disappearance of nausea and/or vomiting

Consistency of disappearance of photophobia

Consistency of disappearance of phonophobia

Consistency of improvement in function at $2 \mathrm{~h}$

Consistency of return to normal function at $2 \mathrm{~h}$

$$
\begin{aligned}
& 73.7 \%\left(28 / 38 ; \mathrm{CI}_{95 \%} 60.0-87.4 \%\right) \\
& 26.3 \%\left(10 / 38 ; \mathrm{CI}_{95 \%} 13.4-43.1 \%\right) \\
& 84.4 \%\left(27 / 32 ; \mathrm{CI}_{95 \%} 71.8-97.0 \%\right) \\
& 45.0 \%\left(9 / 20 ; \mathrm{CI}_{95 \%} 23.2-66.8 \%\right) \\
& 58.3 \%\left(14 / 24 ; \mathrm{CI}_{95 \%} 38.6-78.1 \%\right) \\
& 35.5 \%\left(11 / 31 ; \mathrm{CI}_{95 \%} 18.6-52.3 \%\right) \\
& 40.0 \%\left(10 / 25 ; \mathrm{CI}_{95 \%} 20.8-59.2 \%\right) \\
& 45.7 \%\left(16 / 35 ; \mathrm{CI}_{95 \%} 29.2-62.2 \%\right) \\
& 28.6 \%\left(10 / 35 ; \mathrm{CI}_{95 \%} 13.6-43.5 \%\right)
\end{aligned}
$$

The number of observations analyzed vary by parameter because of the analysis approach in which only patients who had the symptom at baseline in at least one evaluable treatment were included

threshold [33]. Specifically, REN stimulates upper arm peripheral nerves to induce CPM that is evoked by peripheral nociceptive neural activity, which ascends via spinal neurons to the brainstem pain regulation center. Peripheral nociceptive information travels through the ascending somatosensory tract known as the anterolateral system, where it synapses with the brainstem regulation center which includes the rostral ventromedial medulla (RVM), the periaqueductal gray (PAG), and the sub-nucleus reticularis dorsalis (SRD) [32, 34, 35]. The PAG receives input from the ascending fibers as well as other regions (e.g., hypothalamus and amygdala), and communicates with the RVM as well as other medullary nuclei including the SRD [29]. The RVM sends global descending serotonergic projections to the spinal dorsal 
Table 3 Pain relief response at $2 \mathrm{~h}$ post-treatment across multiple treatments

\begin{tabular}{lllll}
\hline 1st treatment (training) & 2nd treatment & 3rd treatment & 4th treatment & 5th treatment \\
\hline $58.8 \%(20 / 34)$ & $50.0 \%(19 / 38)$ & $59.5 \%(22 / 37)$ & $62.5 \%(20 / 32)$ & $63.3 \%(19 / 30)$ \\
$\left(\mathrm{CI}_{95 \%} 40.7-75.4 \%\right)$ & $\left(\mathrm{CI}_{95 \%} 33.4-66.6 \%\right)$ & $\left(\mathrm{CI}_{95 \%} 42.1-75.2 \%\right)$ & $\left(\mathrm{CI}_{95 \%} 43.7-78.9 \%\right)$ & $(\mathrm{CI} 95 \% 43.9-80.1 \%)$ \\
\hline
\end{tabular}

Table 4 Pain-free response at $2 \mathrm{~h}$ post-treatment across multiple treatments

\begin{tabular}{lllll}
\hline 1st treatment (training) & 2nd treatment & 3rd treatment & 4th treatment & 5th treatment \\
\hline $23.5 \%(8 / 34)$ & $26.3 \%(10 / 38)$ & $27.0 \%(10 / 37)$ & $31.3 \%(10 / 32)$ & $23.3 \%(7 / 30)$ \\
$\left(\mathrm{CI}_{95 \%} 10.7-41.2 \%\right)$ & $\left(\mathrm{CI}_{95 \%} 13.4-43.1 \%\right)$ & $\left(\mathrm{CI}_{95 \%} 13.8-44.1 \%\right)$ & $\left(\mathrm{CI}_{95 \%} 16.1-50.0 \%\right)$ & $\left(\mathrm{CI}_{95 \%} 9.9-42.3 \%\right)$ \\
\hline
\end{tabular}

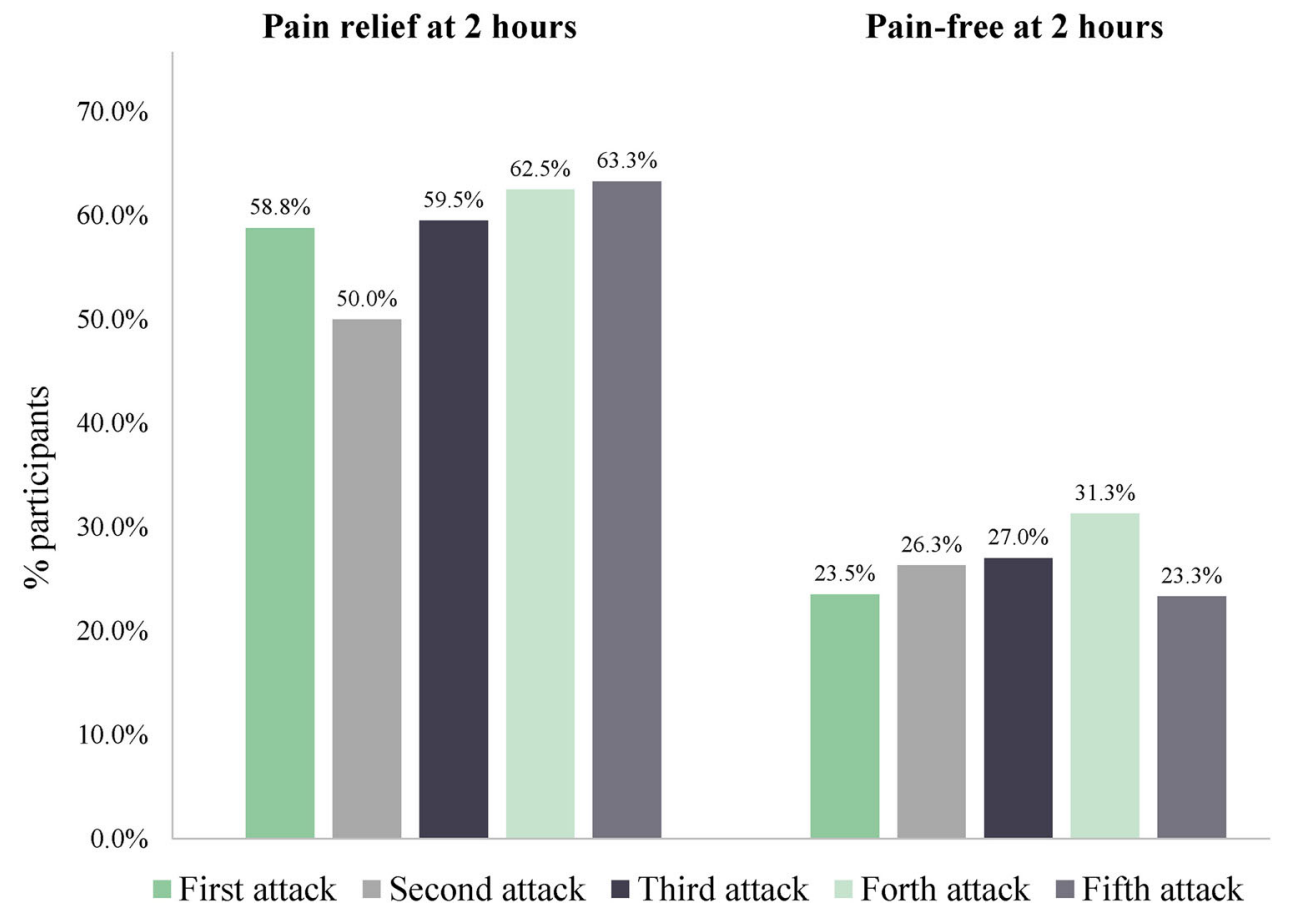

Fig. 4 Pain relief and pain-free responses at $2 \mathrm{~h}$ post-treatment across multiple treatments

horn and trigeminal cervical complex (TCC) that inhibit the information in the ascending projections. In addition, the noradrenergic locus coeruleus (LC) receives inputs from the PAG, communicates with the RVM, and sends descending noradrenergic inhibitory projections [29]. In the context of migraine and the REN device, nociceptive information from the upper arm travels through the ascending somatosensory tract to the brainstem pain regulation center, which sends global descending inhibitory signals by the release of noradrenaline and serotonin (5-HT) [36], which inhibit incoming messages of pain in the TCC that occur during a headache of a migraine attack. 
People with chronic migraine experience at least 15 headache days per month and use their acute migraine medication to treat numerous migraine attacks. Therefore, consistent efficacy and tolerability over multiple migraine attacks are important attributes of acute therapy, especially in this population. Furthermore, consistent efficacy over multiple migraine attacks has been identified by patients as a desirable attribute of acute migraine therapies [28]. The design of the study enabled to evaluate withinsubject consistency of response, which has been suggested to be particularly meaningful clinically because it provides an indication of the individual patient's response to therapy over time [37]. The consistency of the results demonstrates that Nerivio used for a series of migraine attacks was effective and well tolerated across attacks. The consistency of REN is similar to that observed in triptan studies [38, 39], though these comparisons were not obtained in head-to-head trials, and the design, methods, and migraine population (non-chronic vs. chronic) of the studies varied. REN treatment thus may offer a safe and effective non-pharmacological alternative for acute treatment in patients with chronic migraine.

Our findings also demonstrated that REN may eliminate the associated symptoms of migraine: nausea, photophobia, and phonophobia. Improvement in function at $2 \mathrm{~h}$ was also consistent across attacks, with almost half of the patients achieving this outcome. Approximately $30 \%$ of patients returned to normal function at $2 \mathrm{~h}$ in most of their attacks, similar to results reported for lasmiditan, ubrogepant, and rimegepant, although these were reported for a single attack in people without chronic migraine [4, 40, 41].

The International Headache Society guidelines for acute treatment of migraine trials postulate that individuals with chronic migraine or with a history of chronic migraine in the last 12 months should be excluded from efficacy trials for the acute treatment of migraine [22]. Therefore, most studies of acute migraine treatments have not been tested in a trial setting in people with chronic migraine who are characterized with increased likelihood to experience severe pain intensity [42], high disability
[43], less robust response to triptans [44], and high discontinuation rates of prescribed acute treatment due to lack of efficacy [45]. One study that investigated the effects of vagus nerve stimulation in patients with high-frequency episodic migraine and chronic migraine demonstrated a consistent trend toward greater efficacy in patients with episodic migraine compared with patients with chronic migraine [46]. In this context, the efficacy of REN demonstrated in this study is highly encouraging.

The findings of this study also show that the device is safe and well tolerated. The incidence of device-related adverse events over multiple migraine attacks was low (1.8\%), with no device-related serious adverse events. This rate compares favorably to the reported rates for current acute pharmacological treatments [47] and other neuromodulation devices [48]. Importantly, the safety profile of REN is also favorable with respect to new acute pharmacological treatments such as lasmiditan [4], ubrogepant, and rimegepants $[5,6]$.

The current study has several limitations. First, the efficacy results are not placebo controlled. However, over time, with multiple uses, the magnitude of placebo responses is known to drop, so the data on effectiveness in the sheer number of attacks treated over time tends to reflect the actual effectiveness of this device. Indeed, performing the analyses over multiple attacks in a within-subject design is one of the strengths of this study, enabling reducing the bias of novelty and signifying the consistency of the effect. Second, the current study was conducted on a small sample size, though the total number of treated attacks is high. Further studies in a larger sample size are warranted.

\section{CONCLUSIONS}

The current study demonstrates that REN is associated with consistent efficacy in people with chronic migraine. The data also indicate that the incidence of device-related adverse events is very low, providing further support that REN is well tolerated and safe. Therefore, REN may offer a novel alternative for current 
pharmacological and non-pharmacological treatments that combines efficient treatment with minimal side effects. It could be an effective treatment option for achieving consistent relief from migraine symptoms in people with chronic migraine. The meaningful clinical benefits and the favorable safety profile introduce an alternative acute therapy that can be incorporated into usual care even in a highly disabled population and may thus reduce medication use, which may in turn reduce the risk for medication overuse headache.

\section{ACKNOWLEDGEMENTS}

We thank the participants who volunteered for this trial and the investigators and site personnel. We also acknowledge Dr. Nira Koren-Morag for statistical consultation and analyses.

Funding. This study and the journal's Rapid Service Fee were sponsored by Theranica BioElectronics LTD.

Authorship. All named authors meet the International Committee of Medical Journal Editors (ICMJE) criteria for authorship for this article, take responsibility for the integrity of the work as a whole, and have given their approval for this version to be published.

Authorship Contributions. TL, AI, MV, and DH participated in conception and design of the study. HN, JRV, NL, MV, BL and PW acquired the data. TL and MV analyzed the data. $\mathrm{HN}$ and TL drafted the manuscript. All authors made significant contributions to the conception of the analyses, interpretation of the data, and drafting of the manuscript.

Disclosures. Paul Wright has received compensation for consultation from Yeda, Pilot RB, and Vuja De. Hida Nierenberg has received honoraria from Allergan. Nirit Lev participated in advisory boards of Teva Pharmaceutical, EliLilly, and Novartis. Bryan Lewis holds stock in Antares Pharmaceuticals, Allergan Pharmaceuticals, Soliton Inc., Vaxart Inc., and Danaher.
Tamar Lin, Dagan Harris, Maya Vizel, and Alon Ironi are employees of Theranica Ltd. Julio Vieira has nothing to disclose.

Compliance with Ethics Guidelines. The study protocol was reviewed and approved by the local IRB of Meir Medical center and Vassar Brothers Medical Center IRB and was conducted according to Good Clinical Practice and the Declaration of Helsinki guidelines. Before undergoing any study procedures, patients provided written informed consent. The study is registered with ClinicalTrials.gov (NCT04161807).

Data Availability. The data used to support the findings of this study are included within the article.

Open Access. This article is licensed under a Creative Commons Attribution-NonCommercial 4.0 International License, which permits any non-commercial use, sharing, adaptation, distribution and reproduction in any medium or format, as long as you give appropriate credit to the original author(s) and the source, provide a link to the Creative Commons licence, and indicate if changes were made. The images or other third party material in this article are included in the article's Creative Commons licence, unless indicated otherwise in a credit line to the material. If material is not included in the article's Creative Commons licence and your intended use is not permitted by statutory regulation or exceeds the permitted use, you will need to obtain permission directly from the copyright holder. To view a copy of this licence, visit http://creativecommons.org/licenses/bync/4.0/.

\section{REFERENCES}

1. Natoli JL, Manack A, Dean B, et al. Global prevalence of chronic migraine: a systematic review. Cephalalgia. 2010;30(5):599-609. https://doi.org/ 10.1111/j.1468-2982.2009.01941.x.

2. Schwedt TJ. Chronic migraine. BMJ. 2014. https:// doi.org/10.1136/bmj.g1416(Epub ahead of print). 
3. Silva A, Tepper SJ. Acute treatment of migraines. CNS Drugs. 2012;26:823-39.

4. Goadsby PJ, Wietecha LA, Dennehy EB, et al. Phase 3 randomized, placebo-controlled, double-blind study of lasmiditan for acute treatment of migraine. Brain. 2019;142:1894-904.

5. Lipton RB, Dodick DW, Ailani J, et al. Effect of ubrogepant vs placebo on pain and the most bothersome associated symptom in the acute treatment of migraine: the ACHIEVE II randomized clinical trial. JAMA. 2019;322:1887-988.

6. Croop R, Goadsby PJ, Stock DA, et al. Efficacy, safety, and tolerability of rimegepant orally disintegrating tablet for the acute treatment of migraine: a randomised, phase 3 , double-blind, placebo-controlled trial. Lancet. 2019;394:737-45.

7. De Felice M, Ossipov MH, Porreca F. Update on medication-overuse headache. Curr Pain Headache Rep. 2011;15:79-83.

8. Meng ID, Dodick D, Ossipov MH, et al. Pathophysiology of medication overuse headache: insights and hypotheses from preclinical studies. Cephalalgia. 2011;31:851-60.

9. Barloese M, Petersen A, Stude P, et al. Sphenopalatine ganglion stimulation for cluster headache, results from a large, open-label European registry. J Headache Pain. 2018;19:6.

10. Tao H, Wang $\mathrm{T}$, Dong $\mathrm{X}$, et al. Effectiveness of transcutaneous electrical nerve stimulation for the treatment of migraine: a meta-analysis of randomized controlled trials. J Headache Pain. 2018;19:42.

11. Martelletti P, Jensen RH, Antal A, et al. Neuromodulation of chronic headaches: position statement from the European Headache Federation. J Headache Pain. 2013;14:86.

12. Cortese F, Pierelli F, Bove I, et al. Anodal transcranial direct current stimulation over the left temporal pole restores normal visual evoked potential habituation in interictal migraineurs. J Headache Pain. 2017;18:70.

13. Russo A, Tessitore A, Conte F, et al. Transcutaneous supraorbital neurostimulation in "de novo" patients with migraine without aura: the first Italian experience. J Headache Pain. 2015;16:69.

14. Miller S, Matharu M. Non-invasive neuromodulation in primary headaches. Curr Pain Headache Rep. 2017;21:14.

15. Magis D. Neuromodulation in migraine: state of the art and perspectives. Expert Rev Med Devices. 2015;12:329-39.
16. Yarnitsky D, Volokh L, Ironi A, et al. Nonpainful remote electrical stimulation alleviates episodic migraine pain. Neurology. 2017;88:1250-5.

17. Yarnitsky D, Dodick DW, Grosberg BM, et al. Remote electrical neuromodulation (REN) relieves acute migraine: a randomized, double-blind, placebo-controlled, multicenter trial. Headache J Head Face Pain. 2019;59:1240-52.

18. Rapoport AM, Bonner $\mathrm{JH}$, Lin $\mathrm{T}$, et al. Remote electrical neuromodulation (REN) in the acute treatment of migraine: a comparison with usual care and acute migraine medications. J Headache Pain. 2019;20:83.

19. Rapoport AM, Lin T. Device profile of the Nerivio ${ }^{\mathrm{TM}}$ for acute migraine treatment: overview of its efficacy and safety. Expert Rev Med Devices. 2019;0: $1-7$.

20. Marmura MJ, Lin T, Harris D, et al. Incorporating remote electrical neuromodulation (REN) into usual care reduces acute migraine medication use: an open-label extension study. Front Neurol. 2020. https://doi.org/10.3389/fneur.2020.00226(Epub ahead of print).

21. Nir RR, Yarnitsky D. Conditioned pain modulation. Curr Opin Support Palliat Care. 2015;9:131-7.

22. Diener H-C, Tassorelli C, Dodick DW, et al. Guidelines of the International Headache Society for controlled trials of acute treatment of migraine attacks in adults: fourth edition. Cephalalgia. 2019;39:687-710.

23. Lipton RB, Dodick DW, Silberstein SD, et al. Singlepulse transcranial magnetic stimulation for acute treatment of migraine with aura: a randomised, double-blind, parallel-group, sham-controlled trial. Lancet Neurol. 2010;9:373-80.

24. Marmura MJ, Silberstein SD, Schwedt TJ. The acute treatment of migraine in adults: the American Headache Society evidence assessment of migraine pharmacotherapies. Headache J Head Face Pain. 2015;55:3-20.

25. Lipton RB, Hamelsky SW, Dayno JM. What do patients with migraine want from acute migraine treatment? Headache J Head Face Pain. 2002;42: 3-9.

26. Headache Classification Committee of the International Headache Society (IHS). The international classification of headache disorders. Cephalalgia. 2018;38:1-211.

27. Smitherman TA, Burch $\mathrm{R}$, Sheikh $\mathrm{H}$, et al. The prevalence, impact, and treatment of migraine and severe headaches in the United States: a review of 
statistics from national surveillance studies. Headache J Head Face Pain. 2013;53:427-36.

28. Lipton RB, Stewart WF. Acute migraine therapy: do doctors understand what patients with migraine want from therapy? Headache J Head Face Pain. 1999;39:S20-S2626.

29. Ossipov MH, Dussor GO, Porreca F. Central modulation of pain. J Clin Investig. 2010;120:3779-877.

30. Schuh-Hofer S, Fischer J, Unterberg A, Treede RD, Ahmadi R. Spinal cord stimulation modulates descending pain inhibition and temporal summation of pricking pain in patients with neuropathic pain. Acta Neurochir. 2018;160(12):2509-19. https://doi.org/10.1007/s00701-018-3669-7.

31. Yarnitsky D, Arendt-Nielsen L, Bouhassira D, et al. Recommendations on terminology and practice of psychophysical DNIC testing. Eur J Pain. 2010;14: 339-339.

32. Le Bars D, Dickenson AH, Besson J-M. Diffuse noxious inhibitory controls (DNIC). I. Effects on dorsal horn convergent neurones in the rat. Pain. 1979;6:283-304.

33. Lautenbacher S, Roscher S, Strian F. Inhibitory effects do not depend on the subjective experience of pain during heterotopic noxious conditioning stimulation (HNCS): a contribution to the psychophysics of pain inhibition. Eur J Pain. 2002;6: 365-74.

34. Le Bars D, Dickenson AH, Besson J. Diffuse noxious inhibitory controls (DNIC). II. Lack of effect on non-convergent neurones, supraspinal involvement and theoretical implications. Pain. 1979;6: 305 .

35. Bouhassira D, Villanueva L, Bing Z, et al. Involvement of the subnucleus reticularis dorsalis in diffuse noxious inhibitory controls in the rat. Brain Res. 1992;595:353-7.

36. Millan MJ. Descending control of pain. Prog Neurobiol. 2002;66:355-474.

37. Lipton RB, Bigal ME, Stewart WF. Clinical trials of acute treatments for migraine including multiple attack studies of pain, disability, and health-related quality of life. Neurology. 2005;65:S50-58.

38. Dahlof CGH, Lipton RB, McCarroll KA, et al. Within-patient consistency of response of rizatriptan for treating migraine. Neurology. 2000;55:1511-6.

39. Lipton R, Dodick D, Adelman J, et al. Consistency of response to sumatriptan/naproxen sodium in a placebo-controlled, crossover study. Cephalalgia. 2009;29:826-36.

40. Dodick DW, Lipton RB, Ailani J, et al. Ubrogepant, an acute treatment for migraine, improved patientreported functional disability and satisfaction in 2 single-attack phase 3 randomized trials, ACHIEVE I and II. Headache J Head Face Pain. 2020;60: 686-700.

41. Lipton RB, Croop R, Stock EG, et al. Rimegepant, an oral calcitonin gene-related peptide receptor antagonist, for migraine. N Engl J Med. 2019;381: 142-9.

42. Blumenfeld AM, Varon SF, Wilcox TK, et al. Disability, HRQoL and resource use among chronic and episodic migraineurs: results from the International Burden of Migraine Study (IBMS). Cephalalgia. 2011;31:301-15.

43. Silberstein SD, Lee L, Gandhi K, et al. Health care resource utilization and migraine disability along the migraine continuum among patients treated for migraine. Headache J Head Face Pain. 2018;58: 1579-92.

44. Lipton RB, Chu MK. Conceptualizing the relationship between chronic migraine and episodic migraine. Expert Rev Neurother. 2009;9:1451-4.

45. Lipton RB, Hutchinson S, Ailani J, et al. Discontinuation of acute prescription medication for migraine: results from the chronic migraine epidemiology and outcomes (CaMEO) study. Headache J Head Face Pain. 2019;59:1762-72.

46. Barbanti P, Grazzi L, Egeo G, et al. Non-invasive vagus nerve stimulation for acute treatment of high-frequency and chronic migraine: an open-label study. J Headache Pain. 2015;16:61.

47. Thorlund $\mathrm{K}$, Toor $\mathrm{K}, \mathrm{Wu} \mathrm{P}$, et al. Comparative tolerability of treatments for acute migraine: a network meta-analysis. Cephalalgia. 2017;37:965-78.

48. Magis D, Sava S, d'Elia TS, et al. Safety and patients' satisfaction of transcutaneous supraorbital neurostimulation (tSNS) with the Cefaly ${ }^{\circledR}$ device in headache treatment: a survey of 2,313 headache sufferers in the general population. J Headache Pain. 2013;14:95. 